\title{
Analytical and Experimental Study on Gyroscopic Power Generator with Power Feedback
}

\author{
Hiroshi Hosaka* and Yuki Tajima \\ Department of Human and Engineered Environment, Graduate School of Frontier Sciences, \\ the University of Tokyo, 5-1-5 Kashiwa-no-ha, Kashiwa-city, Chiba 277-8563, Japan
}

(Received February 28, 2020; accepted June 30, 2020)

Keywords: energy harvesting, gyroscope, power generator, vibration, positive feedback

A gyroscopic power generator that self-accelerates its spinning velocity via positive power feedback is developed. In our previous study, a power of $1.8 \mathrm{~W}$ was generated for a gyro-generator, but an external power source was necessary to drive the spinning motor. In this study, the power generated by the precession movement of a flywheel (FW) is applied to the spinning motor. This accelerates the spinning velocity by boosting the feedback voltage. A mathematical model of the generator is presented. Then, the relationships among spin acceleration, generated power, and boost ratio are studied using approximate solutions and numerical analyses. Finally, the validity of the theoretical results is verified by experiment.

\section{Introduction}

Internet of Things devices are utilized in many fields. Energy supply is the largest problem in portable applications. To solve this problem, energy harvesting techniques have been studied. ${ }^{(1-3)}$ Vibration harvesters have been extensively studied because vibration is ubiquitous and contains much energy. Conventional generators use the inertial force of a simple pendulum. The inertial force, and thus the power, of low-frequency vibration is very small. The power output of a wristwatch generator, ${ }^{(4)}$ for example, is $10 \mu \mathrm{W}$. In other wearable generators, the power output is $10 \mathrm{~mW}$ at most. ${ }^{(5-7)}$

To increase inertial force at low frequencies, generators that use the gyroscopic effect have been studied. They increase the angular momentum of the vibrating mass by spinning it at a high angular velocity. There are two types of gyro-generator, namely, those driven by a motor and friction. Friction-driven gyro-generators, invented by Mishler ${ }^{(8)}$ in 1973, have been commercialized as a wrist training tool. A flywheel (FW) is rotated via a friction force caused by precession, producing a power of about $0.1 \mathrm{~W} .{ }^{(9,10)}$ However, such gyro-generators can operate only under vibration that is synchronized with the precession cycle. Although theoretical studies on stabilization have been conducted, ${ }^{(11,12)}$ existing devices do not work under arbitrary vibration. Motor-driven gyro-generators rotate a FW by means of a motor. They work under arbitrary vibration. This type of gyro-generator was invented by Norden ${ }^{(13)}$ in

*Corresponding author: e-mail: hosaka@edu.k.u-tokyo.ac.jp

https://doi.org/10.18494/SAM.2020.2846 
1917 for application as gyrostabilizers for ships. Recently, Kanki et al. ${ }^{(14)}$ fabricated a prototype of a wave energy converter (WEC) that has two FWs $(2.7 \mathrm{t}$ each) and generates $20 \mathrm{~kW}$. Bracco et al. ${ }^{(15)}$ also fabricated a WEC with a $200 \mathrm{~kg} \mathrm{FW}$. This type of gyro-generator is very large because the gyro torque, electromagnetic induction, and coil resistance strongly depend on size. Miniaturization greatly reduces the generated power and makes it difficult to harvest motor spin power. The authors previously developed a motor-driven gyro-generator, whose FW is $100 \mathrm{~mm}$ in diameter with a power output of $1.8 \mathrm{~W}^{(16)}$ To achieve this, precession movement stabilization by a precession spring and an electromechanical conversion efficiency increase, achieved by using a high-performance motor and a speed-increasing gear with a ratio of 100 , were applied. In this generator, however, the FW spinning energy was supplied by an external power supply and the spin velocity was constant. Theoretical analyses of the optimization of magnetic damping, ${ }^{(17)}$ the nonlinear behavior of gyros, ${ }^{(18)}$ and the application of gyro-generators to underwater autonomous vehicles ${ }^{(19)}$ have been conducted. However, the spin velocity in these applications is constant. Spin velocity variation caused by a self-power supply has not been researched.

In this study, a gyro-generator that is powered by harvested energy is developed. In this generator, the generated power accelerates the spin velocity and increases the gyro effect, which in turn increases the output power, creating a positive feedback system. The spin velocity increases until the generated voltage reaches the level of the countervoltage of the spinning motor. It is desirable to maximize spin velocity and acceleration. To achieve this, mechanical and electrical equations for the gyro-generator are obtained. The relationships among time, spin velocity, generated power, and efficiency are clarified using approximate solutions and numerical analyses. It is shown that spin velocity is efficiently increased by increasing the boost ratio as the spin velocity increases. Finally, the validity of the theoretical results is verified experimentally using an apparatus with a FW (diameter: $100 \mathrm{~mm}$ ) rotating up to $650 \mathrm{rpm}$.

\section{Nomenclature}

$\boldsymbol{e}_{x}, \boldsymbol{e}_{y}, \boldsymbol{e}_{z}, \boldsymbol{e}_{Y}:$ unit vectors in $x, y, z$, and $Y$ directions

$E_{C}$ : work done by the viscous damper $C$ in one cycle

$E_{s}$ : work done to the FW spin in one cycle

$E_{z}$ : work done by the $\mathrm{FW}$ precession in one cycle

$\boldsymbol{J}_{f w}, J_{f x}, J_{f z}$ : inertia tensor and principal moment of inertias of FW

$\boldsymbol{J}_{i g}, J_{i z}$ : inertia tensor and principal moment of inertia of inner gimbal part

$J_{o g}$ : inertia tensor of outer gimbal part

$I_{g}, I_{s}$ and $V_{g}, V_{s}$ : currents and voltages of generating and spinning motors

$\tau_{g m}, \tau_{s m}$ and $K_{t g}, K_{t s}$ : torques and torque constants of generating and spinning motors

$k_{p}$ : spring constant of precession spring

$n_{p}, n_{s}$ and $\tau_{p g}, \tau_{s g}$ : gear ratios and torques of precession and spin gears

$N$ : boost ratio

$R_{g}, R_{s}$ : terminal resistances of generating and spinning motors

$V_{F}$ : voltage drop across diode

$V_{r}, I_{r}$ : output voltage and current of rectifier 
$x, y, z$ : coordinate system fixed to inner gimbal

$x^{\prime}, Y, z$ : coordinate system fixed to outer gimbal

$X, Y, Z$ : coordinate system fixed to inertial space

$\eta_{p}, \eta_{s}$ : efficiencies of precession and spin gears

$\theta_{Y}, \theta_{Y o}$ : input angle and amplitude

$\theta_{z}, \theta_{z o}$ : precession angle and amplitude

$\theta_{z m a x}$ : upper limit of precession angle

$\tau_{s}, \tau_{z}$ : torques of $\mathrm{FW}$ spin and inner gimbal precession

$\tau_{s b}$ : torque applied from spin gear to FW shaft

$\tau_{g m f}, \tau_{s b f}$ : friction torques of generating motor and spin bearing

$\omega_{i}$ : excitation frequency

$\boldsymbol{\omega}_{f w}, \boldsymbol{\omega}_{i g}, \boldsymbol{\omega}_{\text {og }}$ : angular velocity vectors of $\mathrm{FW}$, inner gimbal, and outer gimbal

$\omega_{g m}, \omega_{s m}$ : angular velocities of generating and spinning motors

$\omega_{S}, \omega_{Y}, \omega_{z}$ : spin angular velocity of $\mathrm{FW}$, pitching angular velocity of inner gimbal, and precession angular velocity of outer gimbal

\section{Configuration of Gyro-generator and Electrical and Mechanical Equations}

\subsection{Configuration of generator}

The configuration of the generator is shown in Fig. 1. The generator consists of a FW, spinning and generating motors, spin and precession gears, a precession spring, a rectifier, a booster, and outer and inner gimbals. The operation sequence is as follows. (1) The FW is rotated by a spinning motor. The initial velocity is given by a battery charged in advance.



Fig. 1. Configuration of gyro-generator. 
(2) A pitching vibration is applied to the outer gimbal by an external torque. (3) The FW begins precession owing to the gyro effect. (4) The precession velocity is increased by the gear. (5) Electrical power is generated by the generating motor. (6) The generated electricity is rectified, and the voltage is boosted and applied to the spinning motor. By this cycle, the spin angular velocity of the FW, the gyro effect, the precession amplitude, and the power generation increase. This is a positive feedback system whose power source is the input vibration energy. The generated electricity is stored as FW kinetic energy.

As the spin angular velocity increases, the countervoltage of the spinning motor increases. When the countervoltage reaches the level of the generated voltage, the flow of current to the spinning motor stops. Thus, voltage boosting is necessary to increase the spin velocity. If the boost ratio is excessively large, however, the current of the generating motor will increase, the voltage drop caused by the generating motor resistance will increase, and thus the input voltage to the booster will drop. In addition, the current of the spinning motor and thus the spin torque will decrease. When the motor torque is less than the bearing friction torque, the spin velocity will drop. Therefore, there exists an optimal boost ratio.

In gyroscopes, the gyro torque is generally maximum when the precession angle is $0^{\circ}$ (the position of the inner gimbal shown in Fig. 1) and when the torque is 0 at $90^{\circ}$. These positions are unstable and stable equilibria, respectively. Thus, when the inner gimbal moves freely, it moves to the $90^{\circ}$ position and the gyro torque and $\mathrm{FW}$ spin disappear even if vibration is applied to the outer gimbal. To avoid this, a torsion spring is attached to the precession shaft to maintain the precession angle near $0^{\circ}$.

\subsection{Assumptions in analysis}

Inertial force is considered for the FW, inner and outer gimbals, and motors. Friction is considered for the precession and spin gears, spin bearing, and generating motor, all of which have large loads and high angular velocities. The gear friction is represented by a constant efficiency for the transmitted power. The bearing friction is represented by a linear equation for the angular velocity. The motor friction is a constant torque. The electromechanical characteristics of the motors are represented by torque constants and terminal resistances. The inductances of the motors are neglected. The rectifier consists of a diode bridge, where the forward voltage drop of the diodes is considered. The booster consists of a diode and an idealized booster that preserves power. The input vibration is a sinusoidal wave and the pitching angle $\theta_{Y}$ is given by Eq. (1). The generator is very light and thus its inertial force does not affect the input angle.

$$
\theta_{Y}=\theta_{Y o} \sin \omega_{i} t
$$

\subsection{Equations of motion for FW, inner and outer gimbals, and spring}

To show the three-dimensional rotation of the rotor, we use the three coordinates shown in Fig. 2. The coordinate system fixed to the inertial space is called the $X Y Z$ system, where 


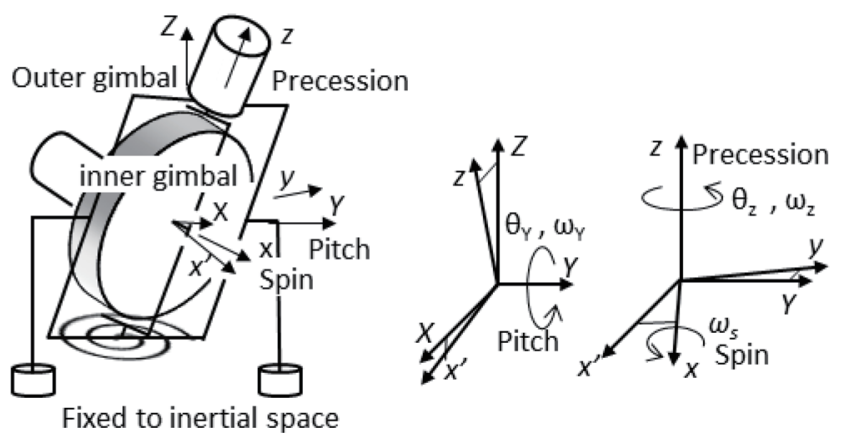

Fig. 2. Coordinate systems.

$X$ is the horizontal direction, $Y$ is the pitching axis of the outer gimbal, and $\mathrm{Z}$ is the vertical direction. The coordinate system fixed to the outer gimbal is called the $x^{\prime} Y z$ system, where $x^{\prime}$ is perpendicular to the outer gimbal surface, $Y$ is as defined above, and $z$ is the rotating axis of the inner gimbal. The coordinate system fixed to the inner gimbal is called the $x y z$ system, where $x$ is the FW spin axis, $y$ is the radial direction of the $\mathrm{FW}$, and $z$ is as defined above. The origin of all coordinates is the center of the FW. The pitching angle is denoted as $\theta_{Y}$ and the precession angle is denoted as $\theta_{z}$; the corresponding angular velocities are respectively denoted as $\omega_{Y}$ and $\omega_{z}$. The FW spin angular velocity is denoted as $\omega_{s}$. Note that $\omega_{s}$ is a relative velocity measured from the inner gimbal, whereas $\omega_{Y}$ and $\omega_{z}$ are the absolute velocities measured from the inertial space. The absolute velocity around the $x$-axis, $\omega_{x}$, is $\omega_{s}$ plus the inner gimbal velocity $\omega_{Y} \sin \theta_{z}$.

The body consisting of the outer gimbal and the generating motor is referred to as the outer gimbal part. The body consisting of the inner gimbal and the spinning motor is referred to as the inner gimbal part. The inertia tensors of these bodies and the FW are denoted as $\boldsymbol{J}_{o g}, \boldsymbol{J}_{i g}$, and $\boldsymbol{J}_{f w}$, respectively. The corresponding principal moments of inertia are denoted by adding the suffix of the axis.

The angular velocity vector of the outer gimbal part, $\omega_{\text {og }}$, is the input angular velocity. The angular velocity vector of the inner gimbal part, $\boldsymbol{\omega}_{i g}$, is the sum of $\boldsymbol{\omega}_{\text {og }}$ and the precession angular velocity $\omega_{z} \boldsymbol{e}_{z}$. The angular velocity vector of the FW, $\boldsymbol{\omega}_{f w}$, is the sum of $\boldsymbol{\omega}_{i g}$ and the spin angular velocity $\omega_{s} \boldsymbol{e}_{x}$. The vectors $\boldsymbol{\omega}_{o g}, \boldsymbol{\omega}_{i g}$, and $\boldsymbol{\omega}_{f w}$ are given as follows when expressed in the coordinates fixed to each part.

$$
\begin{gathered}
\boldsymbol{\omega}_{o g}=\omega_{Y} \boldsymbol{e}_{Y} \\
\omega_{i g}=\omega_{Y} \sin \theta_{z} \boldsymbol{e}_{x}+\omega_{Y} \cos \theta_{z} \boldsymbol{e}_{y}+\omega_{z} \boldsymbol{e}_{z} \\
\boldsymbol{\omega}_{f w}=\left(\omega_{Y} \sin \theta_{z}+\omega_{s}\right) \boldsymbol{e}_{x}+\omega_{Y} \cos \theta_{z} \boldsymbol{e}_{y}+\omega_{z} \boldsymbol{e}_{z}
\end{gathered}
$$

Because the coordinate origins are fixed in space, the kinetic energy $T$ of the whole system is given by the sum of the rotation energies of these parts. 


$$
T=\frac{1}{2} \omega_{o g}{ }^{T} \boldsymbol{J}_{o g} \boldsymbol{\omega}_{o g}+\frac{1}{2} \boldsymbol{\omega}_{i g}{ }^{T} \boldsymbol{J}_{i g} \boldsymbol{\omega}_{i g}+\frac{1}{2} \boldsymbol{\omega}_{f w}{ }^{T} \boldsymbol{J}_{f w} \boldsymbol{\omega}_{f w}
$$

The potential energy of the precession spring $U$ is given as

$$
U=k_{p} \theta_{z}^{2} / 2
$$

Because the vertical movement of the gravity centers of all parts is small, the effect of gravity is neglected. By taking the degrees of freedom as $\theta_{z}$ and $\omega_{s}$ and using $T$ and $U$, the Lagrange equations of motion are derived. Furthermore, by assuming $\omega_{s} \gg \omega_{Y}$, the equations of motion of the precession [Eq. (7)] and spin [Eq. (8)] of the FW are given as follows. The first term in Eq. (7) is the gyro torque.

$$
\begin{gathered}
\tau_{z}=-J_{f x} \omega_{S} \omega_{Y} \cos \theta_{z}+\left(J_{i z}+J_{f z}\right) \dot{\omega}_{z}+k_{p} \theta_{z} \\
\tau_{s}=J_{f x}\left(\dot{\omega}_{s}+\dot{\omega}_{Y} \sin \theta_{z}+\omega_{Y} \omega_{z} \cos \theta_{z}\right)
\end{gathered}
$$

\subsection{Equations of feedback circuit}

The precession velocity $\omega_{z}$ and torque $\tau_{z}$ generated in the inner gimbal shaft become the spin torque of the $\mathrm{FW}, \tau_{s}$, after passing through the feedback circuit and mechanical components shown in Fig. 3. The equations of the components are given as follows.

precession gear

$$
\omega_{g m}=n_{p} \omega_{z} \quad \text { (9a) } \quad \tau_{g m}=\eta_{p} \tau_{z} / n_{p}
$$

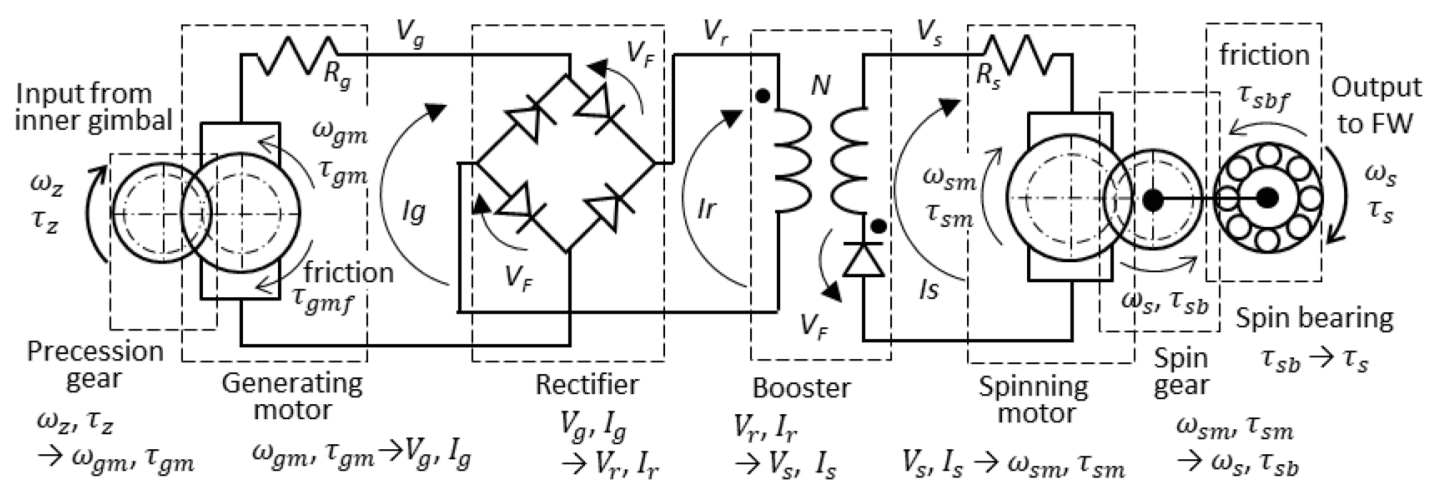

Fig. 3. Feedback circuit and components. 
generating motor

$$
V_{g}=K_{t g} \omega_{g m}-I_{g} R_{g} \quad(10 \mathrm{a}) \quad \tau_{g m}=K_{t g} I_{g}+\tau_{g m f}
$$

rectifier

$$
V_{r}=\left|V_{g}\right|-2 V_{F}
$$

$$
\left|I_{g}\right|=I_{r}
$$

booster

$$
V_{s}=N V_{r}-V_{F}
$$

$$
I_{s}=I_{r} / N
$$

spinning motor

$$
V_{s}=K_{t s} \omega_{s m}+I_{s} R_{s}
$$

$$
\tau_{s m}=K_{t s} I_{s}
$$

spin gear

$$
\omega_{s}=\omega_{s m} / n_{s}
$$

$$
\tau_{s b}=\eta_{s} n_{s} \tau_{s m}
$$

spin bearing

$$
\tau_{s}=\tau_{s b}-\tau_{s b f}
$$

\section{Estimation of Spin Acceleration Using Approximate Analysis}

\subsection{Simplification of equations of generating motor and FW precession}

To estimate the spin acceleration qualitatively, the approximate solutions of equations given in the previous section are used. In this analysis, the mechanical and electrical variables are averaged in a vibration cycle and their long-term characteristics are studied. To simplify the analysis, the following approximations are introduced. Inertia is considered only for the FW. The frictions of the generating motor and spin bearing are included in the efficiencies of the gears. The voltage drop across diodes is neglected. The input frequency is the same as the eigenfrequency of precession. The precession velocity is a sinusoidal function and the spin velocity is constant in one vibration cycle. The velocities are given as below, where $\varphi$ is an unknown constant.

$$
\begin{gathered}
\omega_{z}=\omega_{z o} \sin \left(\omega_{i} t+\varphi\right) \\
\omega_{s}=\text { const }
\end{gathered}
$$

Here, we consider the period $0<\omega_{i} t+\varphi<2 \pi$. The torques $\tau_{z}$ and $\tau_{s}$ are given as follows during the time periods $\alpha<\omega_{i} t+\varphi<\pi-\alpha$ and $\pi+\alpha<\omega_{i} t+\varphi<2 \pi-\alpha$. At other times, they are zero 
because the rectifier and the booster pass only one-way current.

$$
\begin{gathered}
\tau_{z}=\frac{N^{2} K_{t g}{ }^{2} n_{p}{ }^{2}}{\eta_{p}\left(R_{g} N^{2}+R_{s}\right)}\left(\omega_{z}-\operatorname{sgn}\left(\omega_{z}\right) \frac{K_{t s} n_{s} \omega_{s}}{N K_{t g} n_{p} \omega_{z o}}\right) \\
\tau_{s}=\frac{K_{t s} n_{s} \eta_{s} N K_{t g} n_{p}}{R_{g} N^{2}+R_{s}}\left|\omega_{z}-\operatorname{sgn}\left(\omega_{z}\right) \frac{K_{t s} n_{s} \omega_{s}}{N K_{t g} n_{p} \omega_{z o}}\right|
\end{gathered}
$$

The curves of $\omega_{z}, \tau_{z}$, and $\tau_{s}$ in Eqs. (16), (18), and (19), respectively, are shown by continuous lines in Fig. 4. Because they are in phase, $\tau_{z}$ is approximated by a sinusoidal function proportional to $\omega_{z}$, as shown by the dashed line in Fig. 4 .

$$
\tau_{z}=C \omega_{z}
$$

This equation means that the generating motor is approximated by a viscous damper, $C$. The value of $C$ is selected so that the work done by the $\mathrm{FW}$ precession is the same before and after the approximation. The work in one cycle before the approximation is given as

$$
E_{z}=\int_{-\varphi / \omega_{i}}^{(2 \pi-\varphi) / \omega_{i}} \tau_{z} \omega_{z} d t=\frac{4 N^{2} K_{t g}{ }^{2} n_{p}{ }^{2} \omega_{z o}{ }^{2}}{\omega_{i} \eta_{p}\left(R_{g} N^{2}+R_{s}\right)} F(r),
$$

where $r$ is the ratio of the countervoltage of the spinning motor to the boosted voltage of the generating motor and $F$ is the drop of the average electric power caused by the countervoltage and the blocking of the countercurrent in the rectifier and the booster.

$$
r=\frac{K_{t s} n_{s} \omega_{s}}{N K_{t g} n_{p} \omega_{z o}}
$$

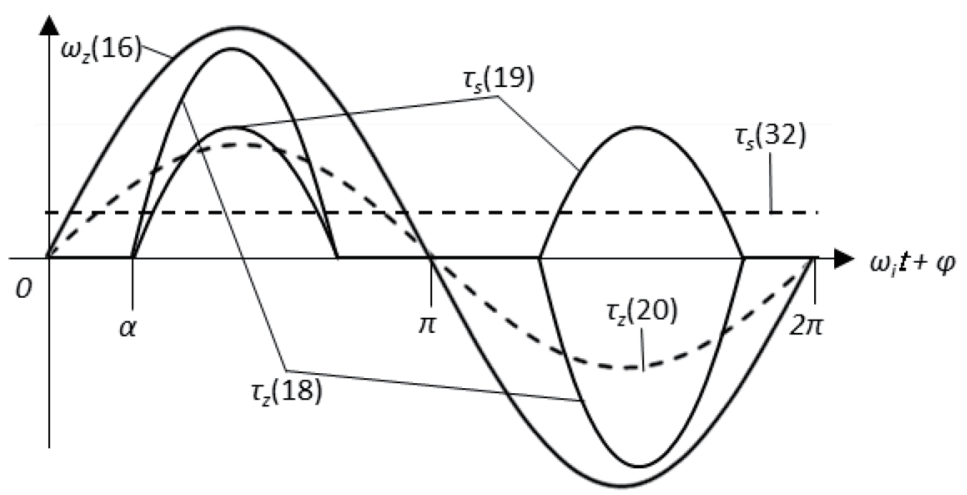

Fig. 4. Curves of simplified torque and velocity. 


$$
F(r)=\int_{\alpha}^{\pi / 2}(\sin x-r) \sin x d x=\frac{\pi}{4}-\frac{\alpha}{2}-\frac{r}{2} \sqrt{1-r^{2}}
$$

The parameter $\alpha$, which is the phase angle when the current of the booster becomes zero, is given as

$$
\alpha=\arcsin r .
$$

The work done by $C$ in one cycle is given as

$$
E_{C}=C \int_{-\varphi / \omega_{i}}^{(2 \pi-\varphi) / \omega_{i}} \omega_{z}^{2} d t=C \frac{\pi \omega_{z o}^{2}}{\omega_{i}}
$$

Thus, $C$ is given as

$$
C=\frac{4 N^{2} K_{t g}^{2} n_{p}^{2}}{\pi \eta_{p}\left(R_{g} N^{2}+R_{s}\right)} F(r)
$$

Next, the equation of motion of precession is simplified. With the assumption $\theta_{z} \ll 1$, Eq. (7) becomes Eq. (27) by using Eq. (20).

$$
J_{f z} \ddot{\theta}_{z}+C \dot{\theta}_{z}+k_{p} \theta_{z}=J_{f x} \omega_{S} \theta_{Y o} \omega_{i} \cos \omega_{i} t
$$

Because the excitation frequency is the same as the eigenfrequency, $\omega_{i}$ and $\theta_{z}$ are given as

$$
\begin{gathered}
\omega_{i}^{2}=\frac{k_{p}}{J_{f z}}, \\
\theta_{z}=\theta_{z o} \sin \omega_{i} t, \\
\theta_{z o}=\frac{J_{f x} \omega_{s} \theta_{Y o}}{C} .
\end{gathered}
$$

Also, $\varphi$ and $\omega_{z o}$ are given as $\varphi=\pi / 2$ and $\omega_{z o}=\theta_{z o} \omega_{i}$, respectively.

\subsection{Simplification of equations of spinning motor and FW spin}

We obtain the average spin torque in one cycle and denote it as $\bar{\tau}_{s}$. With this approximation, the torque variation in one cycle is neglected, but the velocity increment in the cycle is preserved. 


$$
\bar{\tau}_{s}=\frac{\omega_{i}}{2 \pi} \int_{-\varphi / \omega_{i}}^{(2 \pi-\varphi) / \omega_{i}} \tau_{s} d t
$$

Below, we denote $\bar{\tau}_{s}$ as $\tau_{s}$, which is given as

$$
\tau_{s}=\frac{2 N K_{t g} K_{t s} n_{p} n_{s} \eta_{s} \omega_{z o}}{\pi \eta_{p}\left(R_{g} N^{2}+R_{s}\right)} G(r),
$$

where $G$ is the drop in the average voltage caused by the countervoltage and the blocking of the countercurrent.

$$
G(r)=\int_{\alpha}^{\pi / 2}(\sin x-r) d x=\sqrt{1-r^{2}}+r\left(\alpha-\frac{\pi}{2}\right)
$$

The curves of $F, G$, and $G / F$ are shown in Fig. 5. They are monotonically decreasing functions and approach 0 or 1.

Next, the equation of spin motion is simplified. Equation (8) becomes Eq. (34) when integrated in one cycle, where $\Delta \omega_{s}$ is the increment of $\omega_{s}$ in one cycle and $\Delta t$ is the period of the cycle.

$$
\frac{\tau_{s}}{J_{f x}}=\frac{\Delta \omega_{s}}{\Delta t}
$$

The right-hand side of Eq. (34) shows the average acceleration rate of $\omega_{s}$ and can thus approximate $\dot{\omega}_{s}$. Equation (34) can then be rewritten as

$$
\tau_{s}=J_{f x} \dot{\omega}_{s} .
$$

The equations of the generating motor [Eq. (26)], spinning motor [Eq. (32)], FW precession [Eq. (30)], and FW spin [Eq. (35)] are basic equations that represent the long-term characteristics of the gyro-generator.

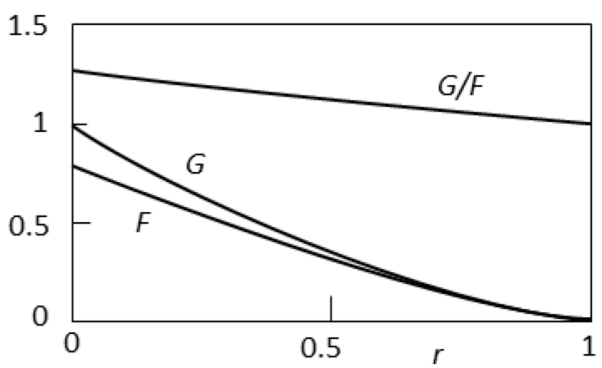

Fig. 5. Countervoltage functions $F$ and $G$. 


\subsection{Qualitative analysis of acceleration using approximate solutions}

By combining the four basic equations, the differential equation for $\omega_{s}$ is obtained as

$$
\dot{\omega}_{s}=\frac{K_{t s} n_{s} \eta_{s} \eta_{p} \omega_{Y_{o}}}{2 N K_{t g} n_{p}} \omega_{s}
$$

The solution is given as below, where $\omega_{s o}$ is determined by the initial conditions.

$$
\omega_{s}=\omega_{s o} \exp \left(\frac{K_{t s} n_{s} \eta_{s} \eta_{p} \omega_{Y o}}{2 N K_{t g} n_{p}} t\right)
$$

The velocity $\omega_{s}$ increases exponentially with time. Note that this solution is valid only under the condition of $\theta_{z o} \ll 1$.

By changing $N$ to $\theta_{z o}$ in Eq. (36) using Eqs. (26) and (30), Eq. (36) becomes Eq. (38). The acceleration increases with $\theta_{z o}$.

$$
\frac{\dot{\omega}_{s}}{\omega_{s}}=\frac{K_{t s} n_{s} \eta_{s} \eta_{p} \omega_{Y o}}{2 K_{t g} n_{p}} \sqrt{\theta_{z o} \frac{4 K_{t g}{ }^{2} n_{p}^{2} F(r)}{J_{f x} \pi \eta_{p} R_{s} \theta_{Y o} \omega_{s}}-\frac{R_{g}}{R_{s}}}
$$

In the actual generator, there is a mechanical restriction on the precession range. The amplitude $\theta_{z o}$ must be smaller than the given upper limit $\theta_{z \max }$, which is $45^{\circ}$ in the experimental apparatus described in Sect. 6. Thus, the acceleration becomes maximum when $\theta_{z o}$ is $\theta_{z \max }$. $\theta_{z o}$ is controlled by $N$. The relationship of $\theta_{z o}$ with $N$ is given as follows using Eqs. (26) and (30). The optimal $N$, which sets $\theta_{z o}$ to $\theta_{z \max }$, increases with $\omega_{s}$.

$$
\frac{1}{N^{2}}=\theta_{z o} \frac{4 K_{t g}^{2} n_{p}^{2} F(r)}{\pi J_{f x} \eta_{p} R_{s} \theta_{Y o} \omega_{s}}-\frac{R_{g}}{R_{S}}
$$

The angular acceleration $\dot{\omega}_{s}$ is given as below from Eqs. (32) and (35).

$$
\dot{\omega}_{s}=\frac{2 N K_{t s} K_{t g} n_{p} n_{s} \eta_{s} \omega_{z o} G(r)}{\pi J_{f x}\left(R_{s}+N^{2} R_{g}\right)}
$$

The acceleration stops at $r=1$ because $G=0$ and $\dot{\omega}_{s}=0$. Then, $\omega_{s}$ becomes maximum and is given as

$$
\omega_{s}=\frac{N K_{t g} n_{p} \omega_{z o}}{K_{t s} n_{s}}
$$


This is the velocity at which the current of the spinning motor becomes 0 owing to the countervoltage.

Next, we obtain the efficiency of the entire system. The input energy is supplied by the input vibration and the output energy is stored as the kinetic energy of the FW. Thus, the efficiency $\eta$ is given by the ratio of the work done to the FW spin, $E_{s}$, to the work done by the FW precession, $E_{C} . E_{S}$ and $\eta$ are given as below from Eqs. (32) and (25).

$$
\begin{gathered}
E_{S}=\tau_{s} \omega_{s} \frac{2 \pi}{\omega_{i}}=\frac{4 N K_{t s} K_{t g} n_{p} n_{s} \eta_{s} \omega_{z o} \omega_{s} G(r)}{\omega_{i}\left(R_{s}+N^{2} R_{g}\right)} \\
\eta=\frac{E_{S}}{E_{C}}=r \eta_{s} \eta_{p}
\end{gathered}
$$

Because $r$ is proportional to $\omega_{s}, \eta$ increases with $\omega_{s}$. Because the maximum $r$ is 1 , the maximum $\eta$ is the product of the efficiencies of the precession and spin gears, $\eta_{s} \eta_{p}$.

The curves of $\omega_{s}, \eta$, and $N$ are shown in Fig. 6. They increase with time. The maximum $\eta$ is $\eta_{s} \eta_{p}$. When $N$ is constant, the rate of increase in $\omega_{s}$ increases with decreasing $N$, and the maximum $\omega_{s}$ increases with $N$. By controlling $N$ so that $\theta_{z o}$ coincides with $\theta_{z \max }$, the maximum $\omega_{s}$ reaches its highest value of $N K_{t g} n_{p} \omega_{z o} / K_{t s} n_{s}$.

\section{Numerical Analysis}

\subsection{Calculation procedure}

In this section, the equations obtained in Sect. 3 are solved without approximation. Equations (9a), (9b)-(15) give the relationships among $\tau_{s}, \tau_{z}, \omega_{s}$, and $\omega_{z}$. By inserting values

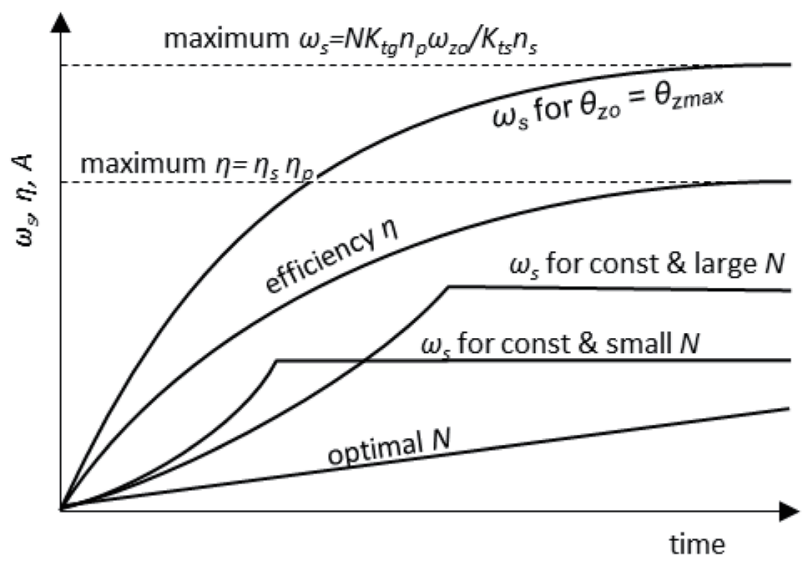

Fig. 6. Curves of $\omega_{s}, \eta$, and $N$ obtained from approximate solution. 
of $\omega_{s}$ and $\omega_{z}$ to these equations, $\tau_{s}$ and $\tau_{z}$ are obtained. By inserting values of $\tau_{s}$ and $\tau_{z}$ to Eqs. (7) and (8), $\omega_{s}$ and $\omega_{z}$ after a short period are obtained. By repeating these procedures, all the parameters are obtained in the time domain. Since Eqs. (9a), (9b)-(15) are algebraic equations, they can be combined into two algebraic equations that directly give $\tau_{s}$ and $\tau_{z}$ from $\omega_{s}$ and $\omega_{z}$. For a future expansion to complicated systems, however, these equations are solved one by one for each component, which enables the simulator to include nonlinear or time-dependent components.

The procedure is shown in Fig. 7. The numbers in the figure are the equation numbers, the symbols are physical quantities transferred between components, and the arrows show the order of calculation. First, initial values are given to $\omega_{z}, \theta_{z}$, and $\omega_{s}$. The initial $\omega_{z}$ is set to 0 to simplify the calculation of $I_{g}$, as mentioned later. Next, by inserting $\omega_{z}$ into Eq. (9a), $\omega_{g m}$ is obtained, and by inserting $\omega_{s}$ into Eq. (14a), $\omega_{s m}$ is obtained. Then, $V_{g}, V_{r}$, and $V_{s}$ are obtained from Eqs. (10a), (11a), and (12a), respectively. In the calculation of Eq. (10a), $I_{g}$ of the previous cycle is used. The initial value of $I_{g}$ is 0 because the rectifier blocks current under the condition of $\omega_{z}=0$. Next, by inserting $V_{s}$ and $\omega_{s m}$ into Eq. (13a), $I_{s}$ is obtained. Then, $I_{r}, I_{g}, \tau_{g m}$, and $\tau_{z}$ are obtained from Eqs. (12b), (11b), (10b), and (9b), respectively. Also, $\tau_{s m}, \tau_{s b}$, and $\tau_{s}$ are obtained from Eqs. (13b), (14b), and (15b), respectively. In the calculation of Eq. (11b), the sign of $I_{g}$ is set to be the same as that of $\omega_{z}$. Since the rectifier transmits energy only from the input (left) to the output (right), signs of $I_{g}$ and $V_{g}$ are the same, and thus the signs of $I_{g}$ and $\omega_{z}$ are the same. Then, Eqs. (7) and (8) are solved using $\omega_{s}, \omega_{z}, \tau_{s}, \tau_{z}, \omega_{Y}$, and $\dot{\omega}_{Y}$ where $\omega_{Y}$ and $\dot{\omega}_{Y}$ are given by Eq. (1), and $\omega_{s}$ and $\omega_{z}$ after a short period is obtained. Then the above procedures are repeated.

To set $N$ to the optimal value, it is necessary to set $\theta_{z o}$ to $\theta_{z \max }$. In the above procedure, however, $\theta_{z o}$ is known only at the moment that $\theta_{z}$ takes a local minimum or maximum value. Even if $\theta_{z o}$ is known, $N$ cannot be obtained directly because the relationship between $N$ and $\theta_{z o}$ [e.g., Eq. (39)] is given only in the approximate analysis. Thus, in this paper, when $\theta_{z}$ takes a local extreme value, $\theta_{\text {zmax }}$ is subtracted from it; if it is positive, $N$ is increased by a certain value and if it is negative, $N$ is decreased. Then, $\theta_{z o}$ approaches $\theta_{z \max }$.

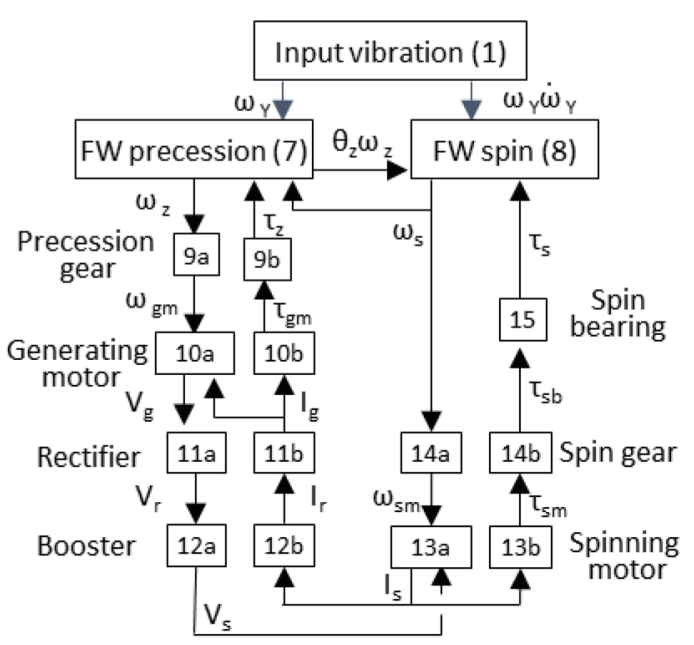

Fig. 7. Calculation flow. 


\subsection{Parameters}

In the calculation, the following conditions are applied. The spinning motor is the coreless DC motor SCL18-3328 (Adamant Namiki Precision Jewel Co., Ltd.) and is called motor A in this study. This motor is small and highly efficient and is used in the experiment described in Sect. 6. For the generating motor, either motor A or the motor NC-256402 (Citizen Chiba Precision Co., Ltd.), which is called motor B, is used. Motor B is larger than motor A and has a higher torque constant and a lower resistance than motor $\mathrm{A}$. The characteristics of the motors provided by the manufacturers are shown in Table 1. Other parameters for the calculation are the same as those for the experiment: $J_{f x}=0.80 \mathrm{gm}^{2}, J_{f z}=0.45 \mathrm{gm}^{2}, J_{i z}=1.3 \mathrm{gm}^{2}, \omega_{i}=2.5 \mathrm{~Hz}, k_{p}=8.2 \mathrm{mNm} / \mathrm{deg}$, $\theta_{z \max }=45^{\circ}, \theta_{Y_{o}}=30^{\circ}$, and initial $\omega_{s}=800 \mathrm{rpm} . \tau_{s b f}$ is given as $\tau_{s b f}=1.5 \times 10^{-5} \omega_{s}+2.6 \times 10^{-4}$, where the units for $\tau_{s b f}$ and $\omega_{s}$ are $\mathrm{Nm}$ and $\mathrm{rad} / \mathrm{s}$, respectively. This is an approximate formula obtained experimentally using our apparatus. The diodes used for the rectifier and the booster are SBM1045VSS (PANJIT Semiconductor), whose forward voltage is $V_{F}=0.25 \mathrm{~V}$ at $0.1 \mathrm{~A}$.

\subsection{Simulation results}

The calculated spin angular velocity $\omega_{s}$ is shown in Fig. 8 for constant boost ratios of $N=1,1.6$, and 5 and the controlled boost ratio. The continuous and dashed lines show the spin velocities obtained from generating motors $\mathrm{A}$ and $\mathrm{B}$, respectively. The numbers indicate $N$. When $N$ is constant and the precession angle $\theta_{z o}$ reaches the upper limit $\theta_{z \max }$ (in this case, $45^{\circ}$ ), the power feedback is stopped and $\omega_{s}$ is set constant. At $t=250 \mathrm{~s}$, the $\omega_{s}$ of motor B reaches $4800 \mathrm{rpm}$ for $N=1.6$ and $9600 \mathrm{rpm}$ for controlled $N$ (data not shown). The rising speed of $\omega_{s}$ is higher for $N=1$ than for $N=1.6$ for both motors A and B. The maximum value of $\omega_{s}$ for $N=1.6$ is larger than that for $N=1$. These characteristics are the same as those obtained from the approximate solution shown in Fig. 6. At $N=5, \omega_{s}$ stalls in both motors A and B. This was not observed in the approximate solution. The stall is caused by the constant spin friction, which is neglected in the approximation and becomes dominant in the low-torque region. When $N$ is very large, the current of the spinning motor is so small that the motor torque is smaller than the friction and $\omega_{s}$ stalls.

Table 1

Characteristics of spinning and generating motors provided by manufacturers.

\begin{tabular}{lcc}
\hline Motor type & A & B \\
\hline$K_{t g}, K_{t s}(\mathrm{mNm} / \mathrm{A})$ & 21 & 32 \\
$R_{g}, R_{s}(\Omega)$ & 28 & 3.75 \\
$\tau_{g m f}(\mathrm{mNm})$ & $9.6 \times 10^{-2}$ & 1.6 \\
$n_{p}, n_{s}$ & $105,3.6$ & 113 \\
$\eta_{p}, \eta_{s}$ & $0.61,0.86$ & 0.73 \\
\hline
\end{tabular}

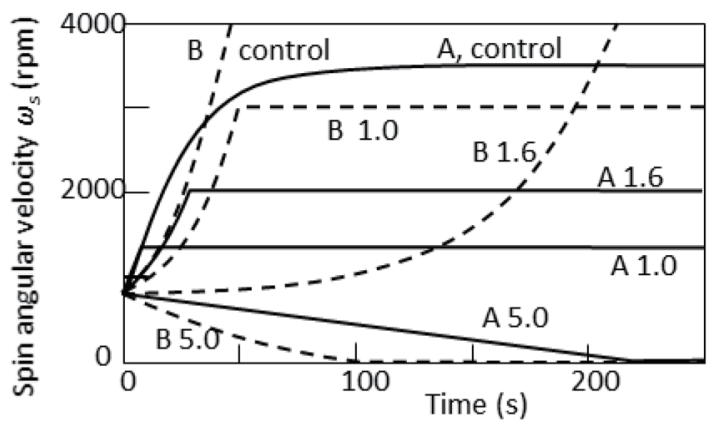

Fig. 8. Effect of boost ratio on spin angular velocity. 
To control the boost ratio in Fig. 8, the initial value of $N$ was set to 0.8 and $\theta_{Y o}$ was obtained every half-period. $N$ was increased or decreased by $2 \%$ in accordance with whether or not $\theta_{Y o}>\theta_{z \max }$ was true. Because $N$ was changed intermittently, this control was considered long-time-interval sampled-data control, not minimum time control of $\omega_{s}$.

Figure 9 shows the change in $N$ for controlled boosting. $N$ increases with time; the trend is the same as that in Fig. 6. $N$ converges to certain values because $\omega_{s}$ becomes constant and the system enters a steady state. The $N$ of motor A rises faster than that of motor B because the $K_{t g}$ of motor A is smaller than that of motor B. The $N$ of motor B is initially constant because it is kept at the initial value until $\theta_{z o}$ reaches $\theta_{z \max }$ and the time to reach $\theta_{z \max }$ is long for motor $\mathrm{B}$ because of its large $K_{t g}$.

\section{Experiment}

The calculated results are verified by experiment. Figure 10 shows the experimental apparatus. The FW, made of aluminum, had a diameter of $100 \mathrm{~mm}$ and a thickness of $30 \mathrm{~mm}$, and spun around a horizontal axis. The input vibration was applied manually via a handle. The rotation of precession was around a vertical axis and the generating motor and the precession gear were fixed on top of the outer gimbal. The input and precession angles were measured by encoders. The spin velocity was measured by a photoreflector placed on the hidden side of the FW. The generating and spinning motors were both motor A. The gear ratios of the precession and spin gears were 22.22 and 3.6, respectively. The ratio $n_{p}$ was set smaller than that in Table 1 because the gears easily broke when $n_{p}$ was around 100. A torsion spring with $k_{p}=8.2 \mathrm{mNm} / \mathrm{deg}$ was fixed below the bottom of the outer gimbal.

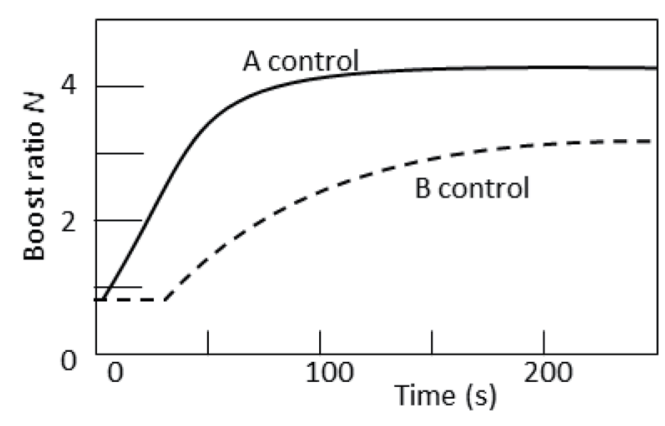

Fig. 9. Time history of boost ratio.

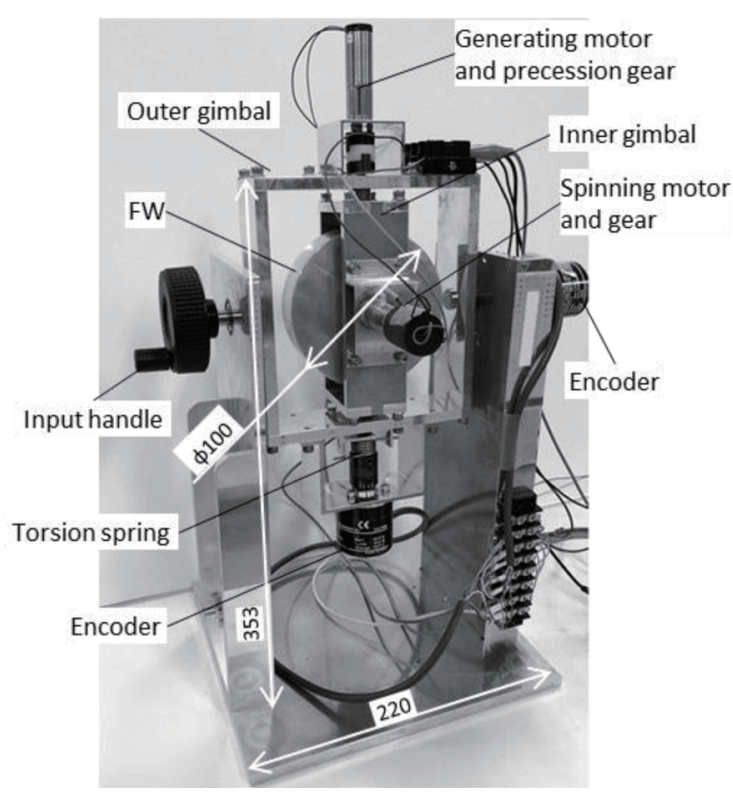

Fig. 10. Experimental apparatus. 


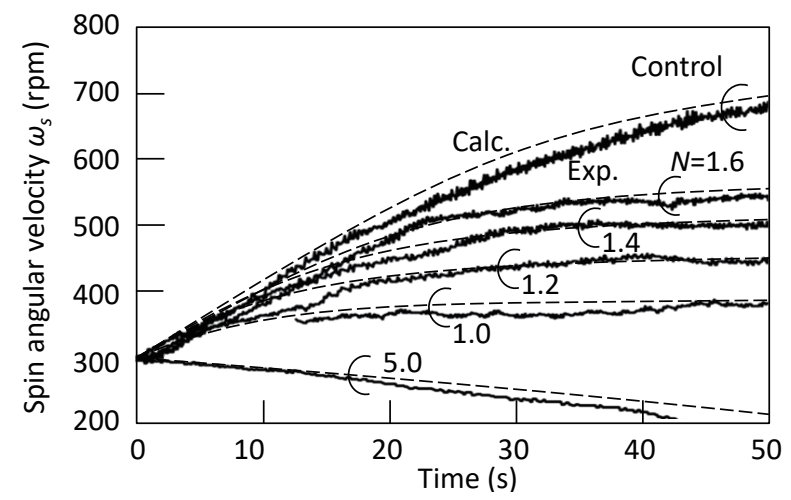

Fig. 11. Experimental and calculated spin angular velocities.

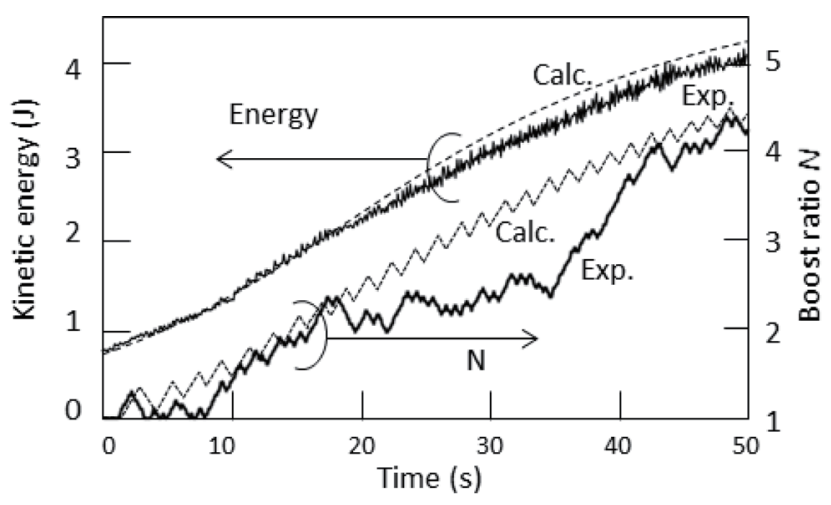

Fig. 12. Experimental and calculated kinetic energies and boost ratios.

An input vibration of $\omega_{i}=2.5 \mathrm{~Hz}$ was applied to the handle with an initial angular velocity of $\omega_{s}=300 \mathrm{rpm}$. When $N$ was constant, $\theta_{Y o}$ was initially set to $8^{\circ}$. After $\omega_{s}$ was increased and $\theta_{z o}$ reached $\theta_{z \max }=45^{\circ}, \theta_{Y o}$ was decreased to keep $\theta_{z o}=\theta_{z \max }$. In the numerical analysis in Sect. 5, feedback was stopped at $\theta_{z o}=\theta_{z \max }$. In the experiment, however, feedback was continued and $\theta_{Y o}$ was decreased to change $\omega_{S}$ in a wide range. When $N$ was controlled, $N$ was increased or decreased by $5 \%$ in accordance with whether $\theta_{z o}$ was larger or smaller than $\theta_{z \max }$, respectively. $\theta_{Y o}$ was set constant to $8^{\circ}$.

The experimental and calculated spin angular velocities are shown in Fig. 11. The $N$ and kinetic energy of the FW are shown in Fig. 12. The continuous and dashed lines are the experimental and calculated results, respectively. In the experiment of controlled $N$, the maximum spin angular velocity was $650 \mathrm{rpm}$, the maximum kinetic energy was $4 \mathrm{~J}$, and the boost ratio changed from 1 to 4.3. In the experiment of constant $N$, the angular velocity increased with time and $N$ when $N$ was less than 1.6 and stalled when $N$ was 5.0. These experimental results agree well with the calculated results. The generated power measured from the slope of the energy was about $0.1 \mathrm{~W}$.

In Fig. 12, both the $N$ and energy from the experiment are smaller than those from the calculation after $18 \mathrm{~s}$. This is because the input vibration was applied manually and $\theta_{Y o}$ was smaller than $8^{\circ}$ at that time. The $N$ from the experiment approached that from the calculation after $35 \mathrm{~s}$ because $\theta_{Y o}$ was corrected to $8^{\circ}$. From 18 to $40 \mathrm{~s}$, the drop in $N$ was larger than that in energy. This is because $N$ was changed by $5 \%$ by every half-period, and thus, $N$ changed slowly and the influence of error was expanded and remained for a long time.

\section{Conclusions}

To realize a small and high-power vibrational generator using the gyro effect, a method of increasing the spin velocity of a FW was proposed. This method boosts the generated voltage and feeds it back to the spinning motor. To analyze spin acceleration characteristics, the electrical and mechanical equations of the components were derived. The inertial forces of the FW and gimbals, the electromechanical transformations of the generating and spinning 
motors, the efficiency of the gears, the friction of the bearing, and the voltage and current transformations of the booster and the rectifier were considered in the equations.

Closed-form approximate solutions were obtained by averaging the equations in one cycle. Strict solutions were also obtained numerically. With these solutions, the spin acceleration was analyzed. It was shown that there is an upper limit of the spin angular velocity. The limit is the angular velocity at which the voltage generated by the generating motor is equal to the countervoltage of the spinning motor. When the precession amplitude is set to the maximum allowable angle of the device, the acceleration becomes maximum. The precession angle is controlled by the boost ratio, which increases with the spin angular velocity.

To confirm the theoretical results, an experimental device with a FW (diameter: $100 \mathrm{~mm}$; thickness: $30 \mathrm{~mm}$ ) was developed. The maximum spin angular velocity was $650 \mathrm{rpm}$, the maximum kinetic energy was $4 \mathrm{~J}$, and the optimal boost ratio changed from 1 to 4.3 . These experimental results agree well with the calculated results.

\section{Acknowledgments}

This research is supported by the A-STEP Program from the Japan Science and Technology Agency, JST.

\section{References}

1 S. Roundy, P. K. Wright, and J. M. Rabaey: Energy Scavenging for Wireless Sensor Networks (Kluwer Academic Publications, Norwell, 2004).

2 H. Kuwano, Eds.: Recent Advances of Energy Harvesting Technologies (CMC Press, Tokyo, 2010) (in Japanese).

3 S. Sudevalayam and P. Kulkarni: IEEE Commun. Surv. Tutorials 13 (2011) 443. https://doi.org/10.1109/ SURV.2011.060710.00094

4 K. Koike: Micro-mechatronics (J. Horological Inst. Japan) 63 (2020) 34 (in Japanese).

5 New power generators harness energy from vibrations, https://www.star-m.jp/eng/topics/3267/ (accessed February 2020).

6 Y. Naruse, N. Matsubara, K. Mabuchi, M. Izumi, and S. Suzuki: J. Micromech. Microeng. 19 (2009) 1. https:// doi.org/10.1088/0960-1317/19/9/094002

7 J. Smilek, Z. Hadas, J. Vetiska, and S. Beeby: Mech. Syst. Signal Process. 125 (2019) 215. https://doi. org/10.1016/j.ymssp.2018.05.062

8 L. Mishler: U.S. Patent 3726146 (1973).

9 Engadget: https://japanese.engadget.com/2006/01/27/manual-power/ (accessed February 2020).

10 T. Ishii, Y. Goto, T. Ogawa, and H. Hosaka: J. Japan Soc. Precision Eng. 74 (2008) 764 (in Japanese).

11 T. Takahashi, J. Iwasaki, and H. Hosaka: J. Robotics Soc. Jpn. 29 (2011) 661 (in Japanese).

12 Z. Zhanga, S. R. K. Nielsenb, and B. Basuc: Procedia Eng. 199 (2017) 1828.

13 C. Norden: U. S. Patent 1,236,204 (1917).

14 H. Kanki, S. Arii, T. Furusawa, and T. Otoyo: Proc. 8th European Wave and Tidal Energy Conf. (EWTWC 2009) 280-283.

15 G. Bracco, A. Cagninei, E. Giorcelli, G. Mattiazzo, D. Poggi, and M. Raffero: Ocean Eng. 120 (2016) 40. https://doi.org/10.1016/j.oceaneng.2016.05.006

16 H. Hosaka, Y. Oonishi, Y. Tajima, and A. Yamashita: Sens. Mater. 31 (2019) 3655. https://doi.org/10.18494/ SAM.2019.2296

17 G. Bracco, M. Canale, and V. Cerone: Control Eng. Practice 96 (2020) 104299. https://doi.org/10.1016/ j.conengprac.2020.104299

18 N. C. Townsend and R. A. Shenoi: Nonlinear Dyn. 72 (2013) 285. https://doi.org/10.1007/s11071-012-0713-7

19 N. C. Townsend and R. A. Shenoi: Auton Robot 40 (2016) 973. https://doi.org/10.1007/s10514-015-9506-4 